\title{
Enhancing the piezopotential from Zinc oxide (ZnO) nanowire usingp-type polymers
}

\author{
Muhammad Yousuf Soomro, Omer Nur and Magnus Willander
}

\section{Linköping University Post Print}

\section{Tweet}

N.B.: When citing this work, cite the original article.

Original Publication:

Muhammad Yousuf Soomro, Omer Nur and Magnus Willander, Enhancing the piezopotential from Zinc oxide ( $\mathrm{ZnO}$ ) nanowire usingp-type polymers, 2014, Materials letters (General ed.), (124), 123-125.

http://dx.doi.org/10.1016/j.matlet.2014.03.047

Copyright: Elsevier

http://www.elsevier.com/

Postprint available at: Linköping University Electronic Press

http://urn.kb.se/resolve?urn=urn:nbn:se:liu:diva-106204 
Enhancing the piezopotential from Zinc oxide $(\mathrm{ZnO})$ nanowire using p-type polymers

\title{
M. Y. Soomro, O. Nur and M. Willander
}

Department of Science and Technology, Campus Norrköping, Linköping University, SE60174 Norrköping, Sweden

\begin{abstract}
We have investigated the effects of different p-type polymer layers on the piezoelectric potential from $\mathrm{ZnO}$ nanowire $(\mathrm{NWs})$ grown on silver $(\mathrm{Ag})$ coated silicon $(\mathrm{Si})$ substrate by the low temperature chemical synthesis method. Piezoelectric measurement was done by a conductive atomic force microscope (AFM). In the case of the poly $(3,4-$ ethylenedioxythiophene-Tosylate (PEDOT-Tos), the output voltage is enhanced by about 95 $\mathrm{mV}$, compared to $18 \mathrm{mV}$ with the poly(3,4-ethylenedioxythiophene) oxidized with poly(4styrenesulfonate) (PEDOT-PSS) layer. The enhancement in the output piezopotential was attributed to the reduction of the screening effect due to free charge carriers. It is suggested that the present method may be one of the best possible alternative ways to improve the piezopotential output from a $\mathrm{ZnO}$ NWs nanogenerators.
\end{abstract}




\section{Introduction}

In the last ten years, significant and rapid development has been made in nanotechnology which facilitates various interesting nanoscale applications. Products like nanorobotics, Microelectromechanical systems (MEMS), implantable biosensors, environmental sensors etc. exploit novel properties of various nanostructures, like nanowires, nanotubes, quantum dots, nanocomposites etc.

The great challenge is this regard is to find a possible alternative approach to power nanodevices with merits like reduced size of nano systems as well as improved performance. Considerable efforts have been directed towards developing a simple and effective route. In this context a great deal of work initially, has been done by Wang et al. [1] who by using atomic force microscope (AFM) demonstrated an approach of $\mathrm{ZnO}$ NWs based piezoelectric nanogenerator $(\mathrm{NG})$, which directly scavenges different forms of mechanical energy like body movement, vibrational energy, etc. from the working environment.

Among the different tetrahedrally coordinated wurtzite compound piezoelectric semiconductors such as $\mathrm{GaN}, \mathrm{CdS}, \mathrm{AlN}$ and $\mathrm{CdSe}, \mathrm{ZnO}$ has been the focus of intensive investigations in recent years because of its highest effective piezoelectric coefficients (9.93 pm/V) [2]. Several one-dimensional (1D) $\mathrm{ZnO}$ nanostructures (e.g., nanowires, nanorods, and nanotubes) have been synthesized under specific growth conditions.

The piezoelectric properties of $\mathrm{ZnO}$ nanostructures, extensively studied in theoretical and experimental work [3-6] and a wide spectrum of novel devices have been fabricated e.g. piezoelectric field effect transistors, the piezoelectric diode, piezoelectric sensors and the piezoelectric nanogenerator $[1,7-9]$.

To take advantage of these NGs into nanodevices applications, it is critical to improve the output potential. Several factors are responsible which limit the output 
piezopotential, e.g., carrier density, growth conditions, and structural morphology of the nanostructures [6]. Screening effect is one of the most unwanted issues which is responsible for the low output potential from a NG. During bending the ZnO NWs, the compressed side of the nanowire preserves the negative piezoelectric potential, while the stretched side with positive potential is partially screened by free electrons [10]. Consequently, to address this issue, many research groups are involved on further enhancing the output piezopotential from NG by minimizing the screening effect [11-12].

PEDOT:PSS is one of the most extensively explored conducting polymers which is commonly used as the anode electrode or hole-transport layer in organic electronics devices [13]. In order to increase the electrical conduction, the polyanion (PSS) can be replaced by small anions, such as Tos, thus preventing the excess of insulating phase generated with polyanions [14].

The objectives of this study was to explore the influence of PEDOT-PSS and PEDOT:Tos as a p-type polymer coating layer on the of piezopotential output from $\mathrm{ZnO} \mathrm{NWs}$ when deflected by AFM.

\section{Experimental Details}

In a typical procedure, $\mathrm{Si}$ substrates were used for the $\mathrm{ZnO}$ NWs growth. A layer of $\mathrm{Ag}$ was deposited on the cleaned substrates by thermal evaporation. The $\mathrm{ZnO}$ NWs studied in this work were prepared by low temperature aqueous chemical solution method. The details of the growth procedure were reported previously [16].

The samples were divided into two groups, one used for the coating with PEDOT-PSS and other used for the coating with PEDOT-Tos. A hole injection layer of PEDOT: PSS was spin-coated at $4000 \mathrm{rpm}$ on top of the $\mathrm{ZnO} \mathrm{NWs}$, then dried at $100{ }^{\circ} \mathrm{C}$ for 1h. PEDOT-Tos is polymerized directly by mixing EDOT monomers and an oxidative solution of iron(iii) tris-ptoluenesulphonate. Baytron-C and Pyridine $\left(\mathrm{C}_{5} \mathrm{H}_{5} \mathrm{~N}\right)$ anhydrous, 
99.8\% were mixed (25:1) and stirred for 1 hour. This solution was spun coated on top of the $\mathrm{Si}$ substrate with $\mathrm{ZnO}$ NWs. The EDOT 3,4-Ethylenedioxythiophene $\left(\mathrm{C}_{6} \mathrm{H}_{6} \mathrm{O}_{2} \mathrm{~S}\right)$ with molecular weight of $142.18 \mathrm{~g} / \mathrm{mol}$ was evaporated by putting a few drops on a glass slide on a hot plate at $60^{\circ} \mathrm{C}$ for about five hours. The sample was then rinsed with Butanol, IPA and DI water.

\section{Results and discussion}

The morphology of the grown $\mathrm{ZnO}$ NWs was characterized with scanning electron microscope (SEM). As shown in Fig. 1 these NWs were aligned vertically to the substrate surface and $300 \mathrm{~nm}$ in diameter and several micrometers in length.

The piezoelectric properties of the $\mathrm{ZnO}$ NWs were characterized by using contact mode of an AFM having a tetrahedral conductive $\mathrm{Si}$ tip coated with platinum $(\mathrm{Pt})$ film under ambient conditions. For the experimental details the reader is directed to reference 16.

The tip scans over the top of the sample surface over an area of about $10 \times 10 \mu \mathrm{m}^{2}$ from top to bottom and a constant force is applied to bent aligned NWs repeatedly and the bending distance along the z-axis was recorded in the topographic AFM scan image as shown in Fig. 2. (a) and (b).

When the tip scans over the surface of the sample the output signal voltage was continuously monitored. During the bending, a strain field produced in individual $\mathrm{ZnO}$ NW resulting polarization of charge within NW, the tensile side of NW possesses positive charges and the compressive side obtains negative charges reference to the ground root of the NW. These polarization charges cannot freely move and cannot recombine without releasing the strain.

When the AFM tip is in contact with the stretched side of the $\mathrm{ZnO} \mathrm{NW}$, the $\mathrm{Pt} / \mathrm{ZnO}$ interface corresponds to a reverse biased Schottky diode. Electrons flow slowly from the grounded 
electrode through the external load to reach the tip, but the electrons cannot pass across the $\mathrm{Pt} / \mathrm{ZnO}$ interface because of the presence of a reverse-biased Schottky barrier at the contact which serves as a gate for blocking the back flow of electrons and little current flows across the interface [16]. This is the main process that creates, separates and preserves the piezoelectric potential. On the other hand, when the AFM tip touches the compressed side of the $\mathrm{ZnO} \mathrm{NW}$, the metal tip/ZnO contact will become a forward biased Schottky contact. Therefore, the output voltage will be increased. This is the discharging process, and the flow of electrons takes place on the expense of the piezoelectric potential from the tip into the NWs. The output piezopotential from the ZnO NWs is shown in the Fig. 3 (a) and (b) from ZnO NWs with PEDOT-PSS and PEDOT-Tos, respectively.

A three-dimensional (3D) plot of the output voltage corresponding to the feedback signal from the scanner is shown in Fig. 4(a). The output voltage observed from a sample with PEDOT-PSS layer reached up to $18 \mathrm{mV}$. In comparison, a 3D plot of the output voltage for ZnO NWs with layer of PEDOT-Tos in fig. 4 (b) showed much higher output potential amplitude with maximum value of about $95 \mathrm{mV}$. The output sharp peaks are corresponding to the maximum deflection of the NWs. By analyzing the positions of the observed peaks in the topography image and the output voltage image, we noticed that the output voltage signal was detected when the tip reached the compressed side of the NW, indicating that the discharge occurs at the end of the tip scan over the NWs. As in every piezoelectric material there are free electrons/holes and those carriers will redistribute due to the electric field established by the polarization so these piezoelectric polarization charges might be screened by the free charge carriers [11].

In this situation presumably a key role played by the PEDOT-PSS and the PEDOT-Tos layers, and holes from those p-type materials made a layer around the NWs surface and reducing the screening effect caused by free charges, some 5 times higher 
piezopotential output is observed when compared $\mathrm{ZnO}$ NWs with a layer of PEDOT-PSS and PEDOT-Tos.

It is believed that this approach may prove a convenient way for the piezoelectric harvesting properties using p-type polymer with a number of advantages. First, this is very cheap and convenient, even more important; this method is potentially useful for large area processing. Secondly, it uses simple catalyst-free, low cost, low temperature method to grow $\mathrm{ZnO}$ NWs. Last, an important feature of this emerging technique possesses potential for scale up, and it is likely to be adaptable for industrial mass production.

\section{Conclusions}

In summary, piezoelectric effect based on $\mathrm{ZnO}$ NWs with different p-type polymer layers was investigated by AFM. A p-type polymer layer around the NWs can enhance of the piezopotential output from NG by the reduction of the screening effect caused by the free charge carriers. It is expected that, this approach support an immense interest in research in the increasing output potential from $\mathrm{ZnO}$ NW based NG. 
Reference

[1] Wang ZL, Song J. Science 2006; 312:242-6

[2] Zhao MH, Wang ZL, Mao SX. Nano Lett 2004; 4: 587-90

[3] Lin YF, Song J, Ding Y, Lu SY, Wang ZL. Adv Mater 2008; 20:3127-30

[4] Hu Y, Zhang Y, Xu C, Lin L, Snyder RL, Wang ZL. Nano lett 2011; 11:2572-7

[5] Xu S, Qin Y, Xu C, Wei Y, Yang R, Wang ZL. Nature nanotech 2010; 5:366-73

[6] Riaz M, Song J, Nur O, Wang ZL, Willander M. Adv Funct Mater 2011; 21:628-33

[7] Fei P, Yeh PH, Zhou J, Xu S, Gao Y, Song J, Gu Y, Huang Y, Wang ZL. Nano Lett; 2009; 9:3435-9

[8] He JH, Hsin CL, Liu J, Chen LJ, Wang ZL. Adv Mater 2007; 19:781-4

[9] Zhou J, Fei P, Gao Y, Gu Y, Liu J, Bao G, Wang ZL. Nano Lett 2008; 8:2725-30

[10] Gao Y, Wang ZL. Nano lett 2009; 9:1103-10

[11] Hu Y, Lin L, Zhang Y, Wang ZL. Adv Mater 2012; 24:110-4

[12] Lee KY, Kumar B, Seo JS, Kim KH, Sohn JI, Cha SN, Choi D, Wang ZL, Kim SW. Nano Lett 2012; 12:1959-64

[13] Groenendaal L, Jonas F, Freitag D, Pielartzik H, Reynolds JR. Adv Mater 2000; 12:48194

[14] Jensen BW, West K. Macromolecules 2004; 37:4538-43

[15] Shao Z, Wen L, Wu D, Zhang X, Chang S, Qin S. Physica E 2010; 43:173-175

[16] Soomro MY, Hussain I, Bano N, Nur O, Willander M. Phys. Status Solidi RRL 2012; 6: 80-82 
Figure captions

Figure 1 (a): SEM image of the Top-view ZnO NWs grown on Si substrate with PEDOTPSS coating.

Figure 1 (b): SEM image of the Top-view ZnO NWs with PEDOT-Tos coating.

Figure 2 (a): The topography image showing the bending distance of the $\mathrm{ZnO}$ NWs arrays with a layer of PEDOT-PSS during the scanning.

(b) Topography image of ZnO NWs with a layer of PEDOT-Tos layer.

Figure 3 (a): Output piezopotential from the $\mathrm{ZnO}$ NWs without PEDOT-Tos layer. The pink circle blue square represents scanning and the resulting output potential.

(b) Output piezopotential from the ZnO NWs with PEDOT-Tos layer. The pink circle blue square represents scanning and the resulting output potential.

Figure 4 (a): Three-dimensional (3D) plot of the output voltage corresponding to the feedback signal from the scanner without PEDOT-Tos layer.

(b) Three-dimensional (3D) plot of the output voltage corresponding to the feedback signal from the scanner with PEDOT-Tos layer. 


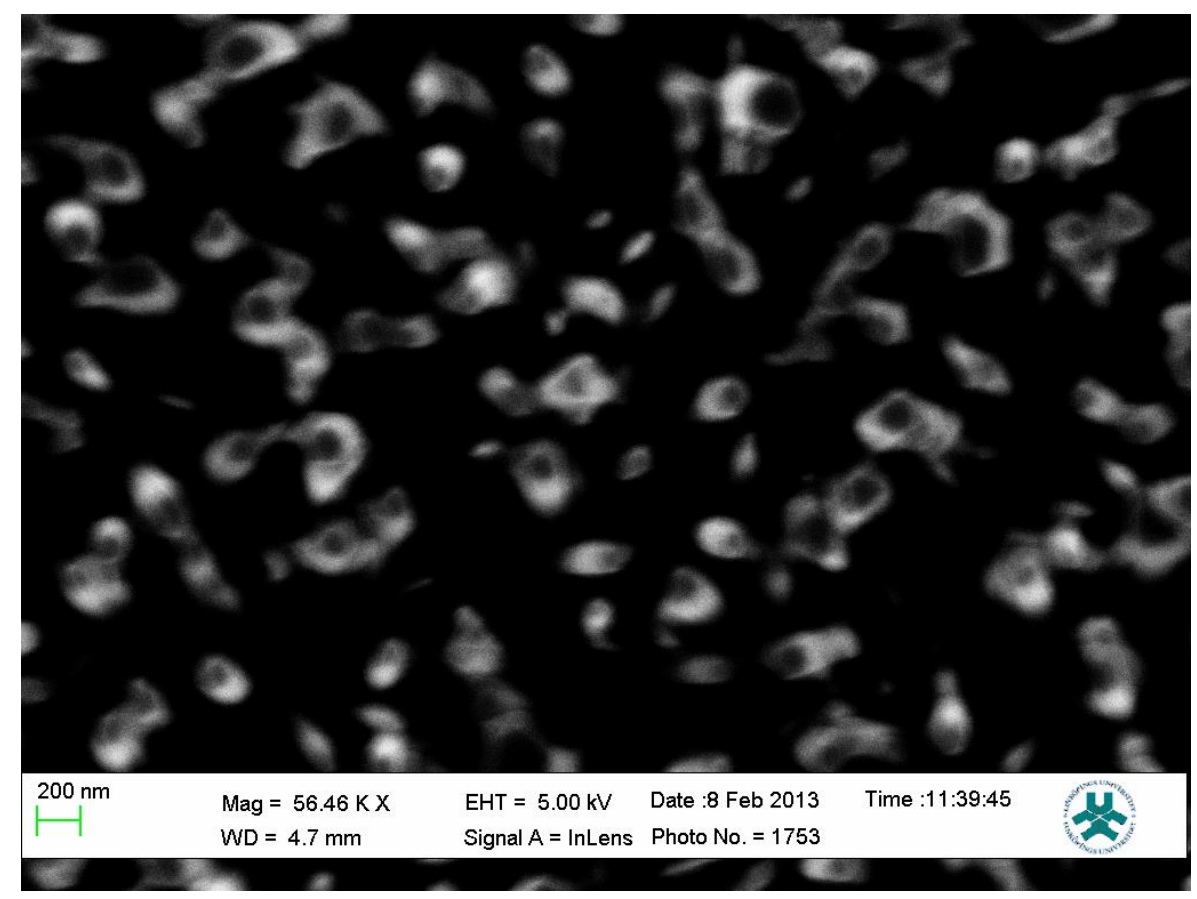

Fig. 1 (a)

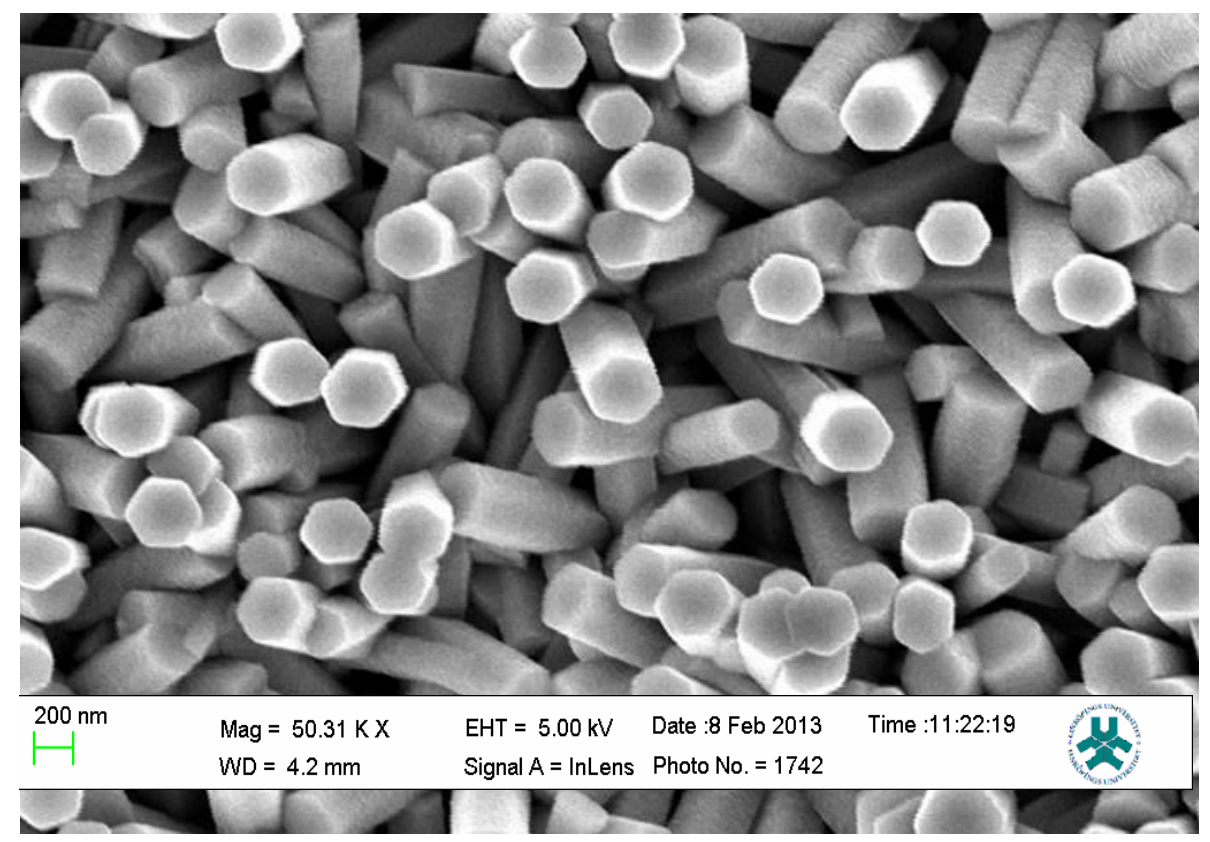

Fig. 1 (b) 


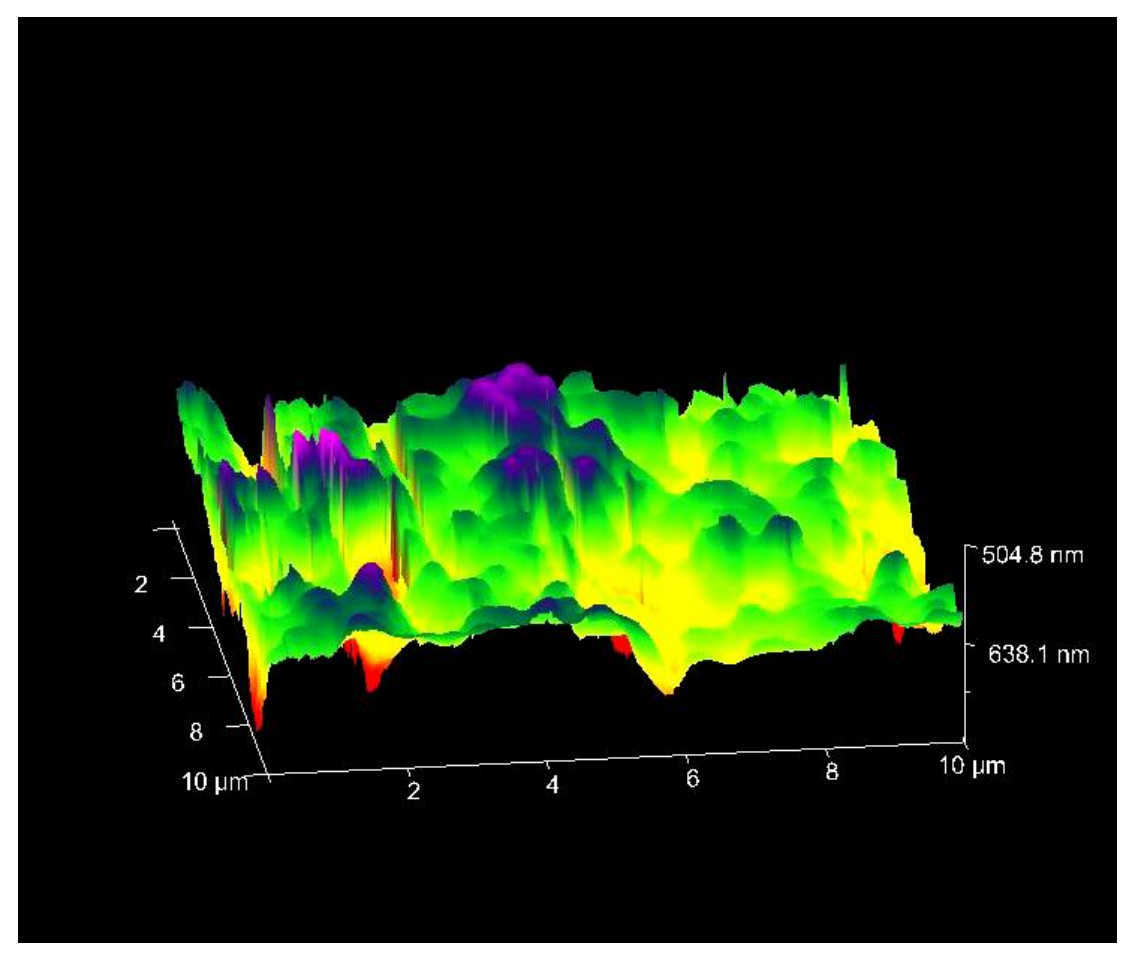

Fig. 2 (a) topography image of $\mathrm{ZnO}$ NWs coated with PEDOT-PSS

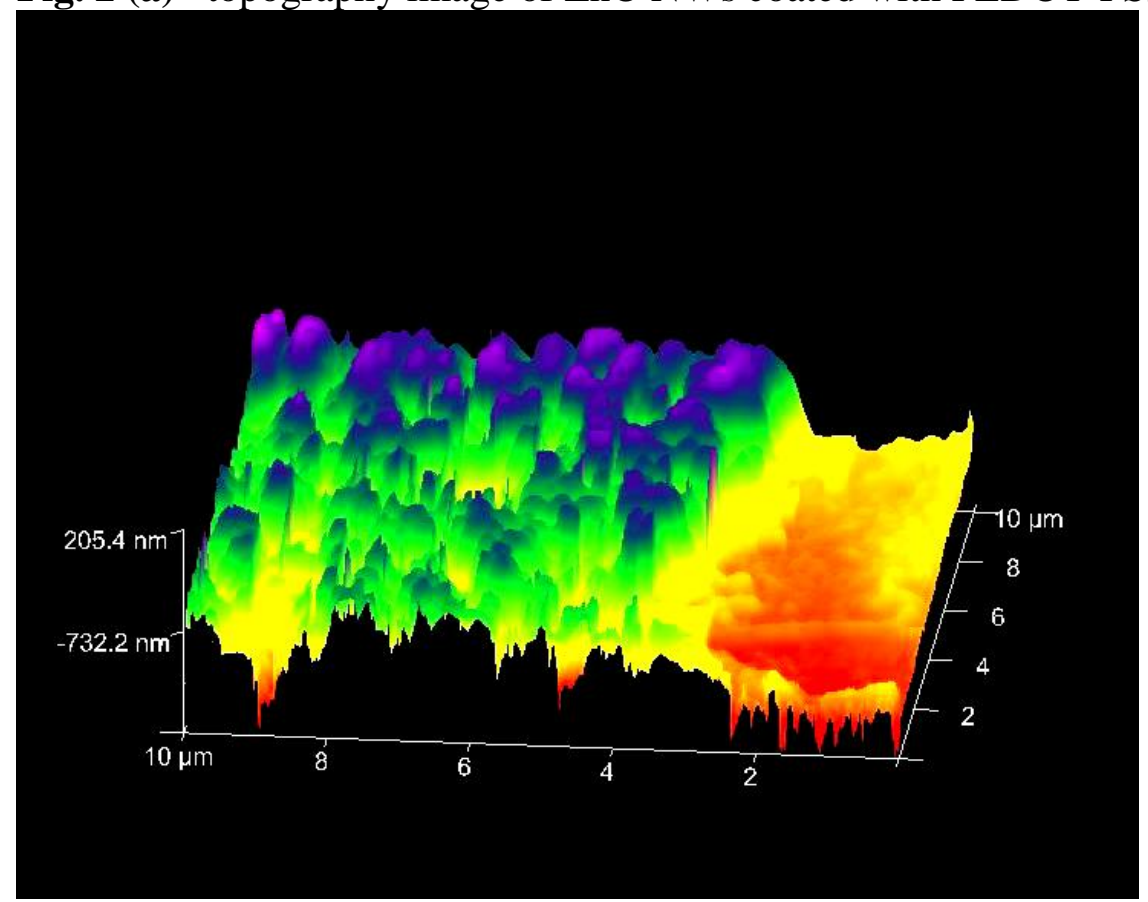

Fig. 2 (b) topography image of $\mathrm{ZnO}$ NWs coated with PEDOT-Tos 


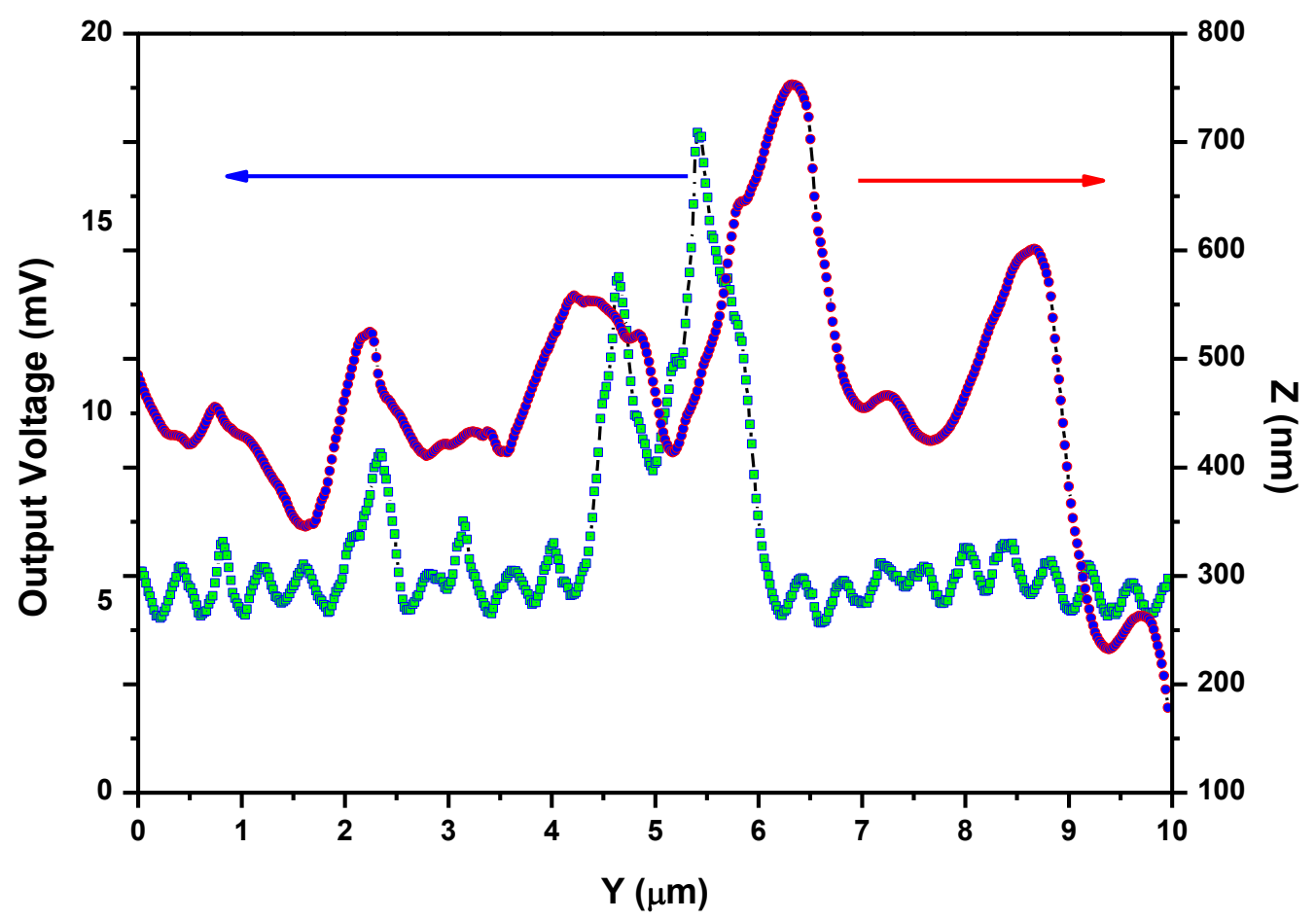

Fig. 3 (a)

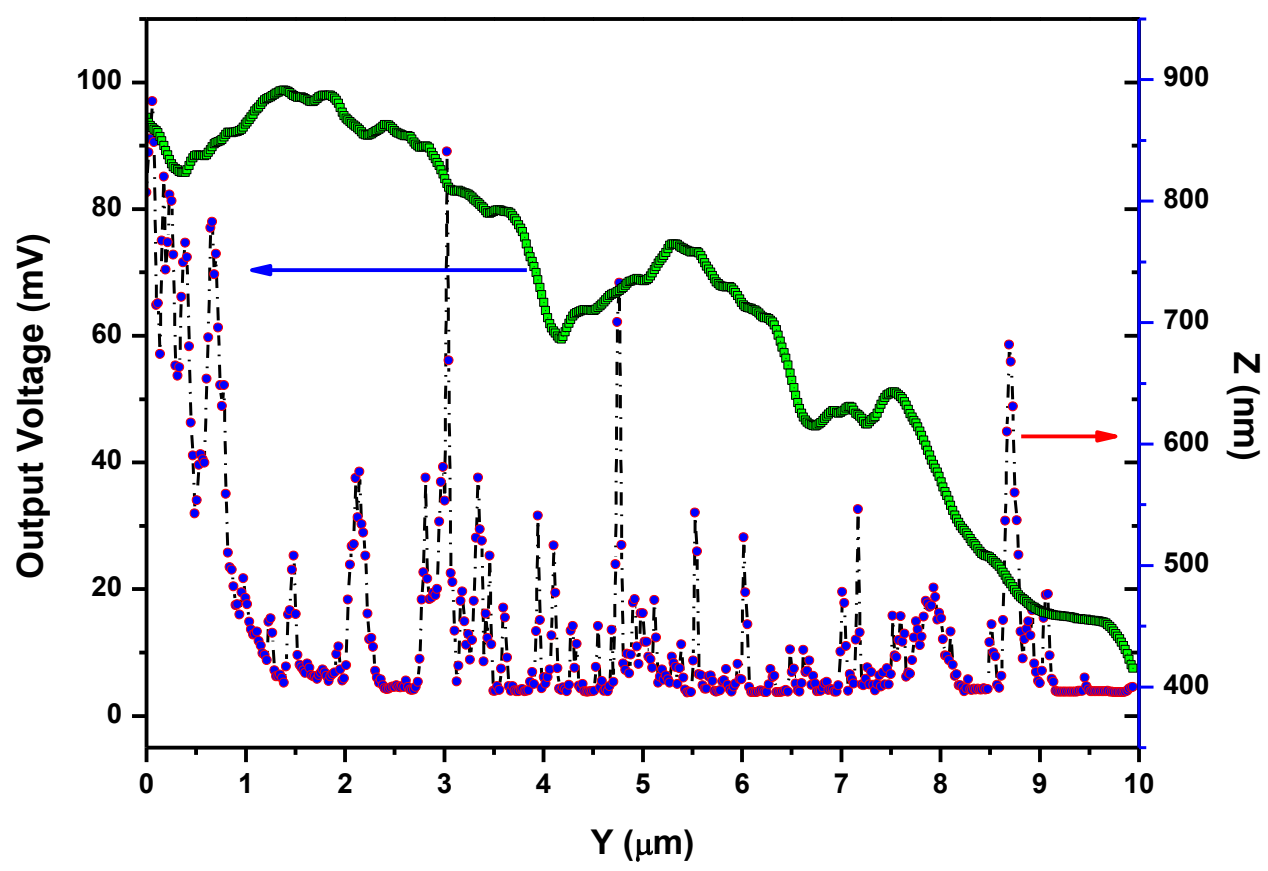

Fig. 3 (b) 


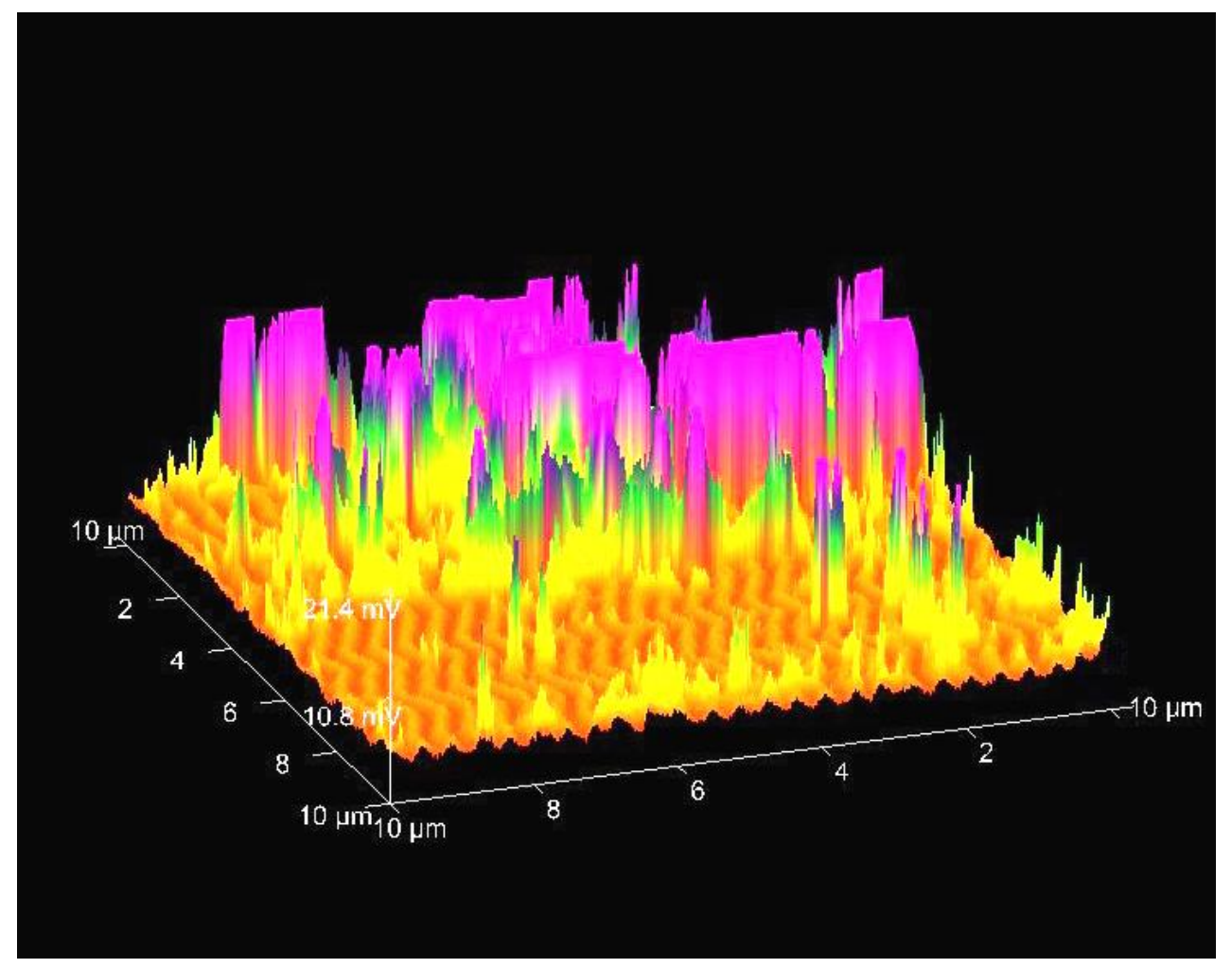

Fig. 4 (a)

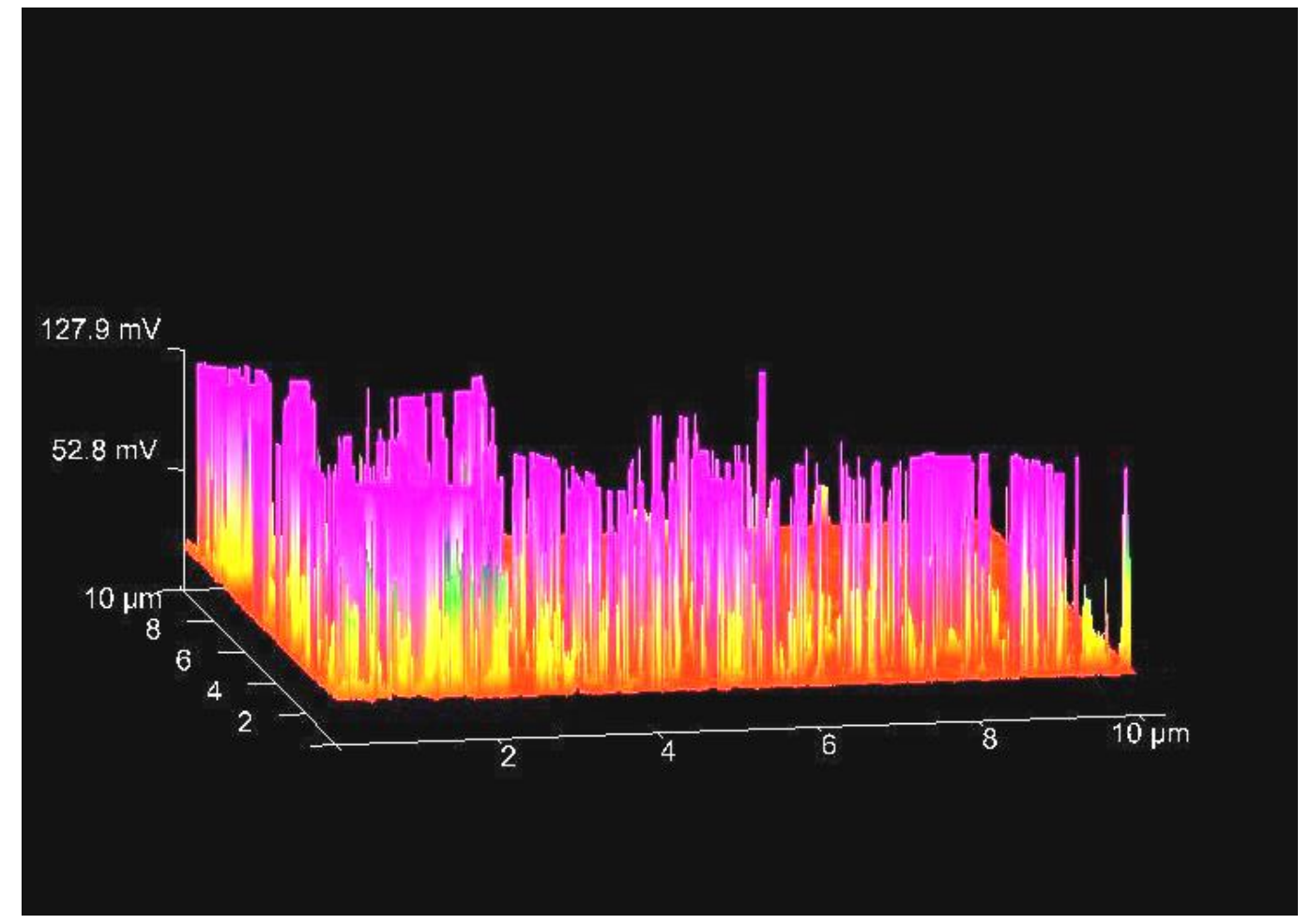

Fig. 4 (b) 